\section{In silico drug design}

\section{By Joanne Kotz, Senior Editor}

Researchers at the University of Dundee and The University of North Carolina at Chapel Hill School of Medicine have created a computational algorithm that mines medicinal chemistry literature to predict new ligands that bind specific combinations of $\mathrm{G}$ protein-coupled receptors. ${ }^{1}$ Ex Scientia Ltd. was spun out of Dundee to commercialize the findings and already has two deals in place related to the screening technology.

Developing a small molecule that binds a predefined combination of targets is at best a time-consuming medicinal chemistry effort and often has proven flat-out impossible.

One major obstacle to designing such drugs is the limited human capacity to sift through the vast accumulation of medicinal chemistry data related to multiple targets and then identify an optimal solution.

A team led by Andrew Hopkins and Bryan Roth reasoned that a computational algorithm might be able to more effectively undertake medicinal chemistry design than a person could and thus could better identify ligands with predetermined polypharmacology, which is the modulation of multiple targets.

Hopkins is chair of medicinal informatics and professor of translational biology at Dundee and founder and managing director of Ex Scientia. Roth is a professor in the Department of Pharmacology at the UNC at Chapel Hill School of Medicine.

First, their team mined data in the ChEMBL public database, which contains compound and activity data extracted from decades of published medicinal chemistry literature. The group used this data to build Bayesian models of ligand activity across 784 human protein targets, including G protein-coupled receptors (GPCRs).

As proof of concept, the researchers decided to study the Alzheimer's disease (AD) drug Aricept donepezil, an acetylcholinesterase (AChE) inhibitor marketed by Pfizer Inc. and Eisai Co. Ltd.

The models predicted that Aricept could also act in vitro on the dopamine $\mathrm{D} 4$ receptor but not the dopamine D2 receptor, two receptors for which there are large amounts of ligand-binding data. Indeed, experimental measurements confirmed that donepezil was a moderately potent D4 receptor inverse agonist and had essentially no activity against the $\mathrm{D} 2$ receptor.
The team next tested if the models could identify new ligands that retained D4 receptor activity, had increased D2 receptor activity and had the ability to penetrate the blood brain barrier (BBB).

The models predicted that eight isoindole-based compounds would have the desired activities. Of those, all eight had activity on both D4 and $\mathrm{D} 2$ receptors. The team tested the most potent compound in mice, and the molecule crossed the BBB.

Although the eight molecules had activity against the desired targets, they also had activity against targets likely to confer side effects. For example, the molecules blocked some adrenoreceptors, which is known to result in hypotension.

Thus, the team next sought to predict ligands with a more complicated combination of targets they wanted to hit and ones they wanted to avoid.

The models suggested that some benzolactam-based molecules would have activity against three particular dopamine receptors and a serotonin receptor but not against select adrenoreceptors. These predictions were confirmed experimentally.

Overall, 800 ligand-target interactions were predicted, of which about 600 (75\%) were successfully confirmed experimentally.

Results were published in Nature.

"What we've found is that medicinal chemistry experience can be encoded and mimicked to a degree. We can now move from human judgment to exploiting vast amounts of data and come up with quite sensible-looking chemistry," said Hopkins.

\section{Automating discovery}

The biggest limitation of the computational drug design approach is the need for existing medicinal chemistry data, making it unlikely the method can be applied to new targets.

"The power of this method is that it lets you leverage every bit of published data in a systematic way. You can evaluate all the possibilities in a way that you couldn't in your head. For example, it's been very difficult to design GPCR ligands against multiple targets-they have usually been designed quasi-randomly based on hope and luck," said Jon Mason, senior research fellow at GPCR drug discovery company Heptares Therapeutics Ltd.

Kerry Spear, VP of medicinal chemistry at Sunovion Pharmaceuticals Inc., a subsidiary of Dainippon Sumitomo Pharma Co. Ltd., added, "I think that this paper has the potential of being a seminal paper for the pharmaceutical industry. Interest in polypharmacology has always been clouded by the fact that you have to optimize multiple SARs. The more SARs you have, the harder the process is and the longer it takes. This outlines a framework for optimizing multiple SARs in a systematic way."

Sunovion's internal research focuses on CNS disorders.

Mason said that ligands designed against a particular target are often not assayed against more than a handful of potential off targets until fairly late in development. When the goal is to design a selective ligand, the computational models could be very useful for identifying unanticipated and undesirable off-target effects early in the drug discovery process, which "could improve efficiency up front," he said.

Combining public and in-house company data may be the best use of 


\section{ANALYSIS}

the models, according to Mason. "Local models trained on your own data are even better. A company could update the models overnight with any new data to have an up-to-date activity model for every target," he said.

"There are really two caveats to the method right now," noted Spear. "First, the method doesn't speak to target selection; that's a big unknown. The other caveat is that the process depends on reliable data, and a lot of it. There is the possibility that there are targets you are interested in where there isn't enough data to allow the algorithms to work effectively."

Mason agreed. “The method is very good but not the 'eureka' for everything," he said. "You need to have large datasets for the targets of interest. For new targets with no published data, you can't model and evolve compounds. You also can't explore completely unknown ways of ligands interacting with targets."

Mason said Heptares is "working now mostly on undrugged targets where there are very limited data." In this case, a structure-based approach is preferable for the primary target, he said.

\section{The leading edge}

Hopkins now is working to incorporate structural information into the algorithm. He hopes this will open the method up to identifying ligands against previously unknown targets or ligands that interact at new sites on targets. The team also is pursuing the application of the method to other target classes, such as kinases.

The team is further interested in exploring whether the computational models can be used for lead optimization. "The next challenge is can we more efficiently optimize lead matter? We are working on methods to reduce the number of compounds required to be synthesized in the lead optimization process to increase productivity," said Hopkins.

Hopkins founded Ex Scientia in July 2012. He said the company has "two deals signed and a third in the pipeline." Ex Scientia plans to apply the current algorithm and develop it further through collaborations on projects at the partner companies. Ex Scientia also hopes to develop an internal pipeline of drug candidates designed using the technology platform.

Dundee has filed a patent application on the methodology, which has been licensed to Ex Scientia.

Kotz, J. SciBX 6(3); doi:10.1038/scibx.2013.50

Published online Jan. 24, 2013

\section{REFERENCES}

1. Besnard, J. et al. Nature; published online Dec. 12, 2012; doi:10.1038/nature11691

Contact: Andrew L. Hopkins, University of Dundee, Dundee, U.K. e-mail: a.hopkins@dundee.ac.uk

Contact: Bryan Roth, The University of North Carolina at Chapel

Hill School of Medicine, Chapel Hill, N.C.

e-mail: bryan_roth@med.unc.edu

\section{COMPANIES AND INSTITUTIONS MENTIONED}

Dainippon Sumitomo Pharma Co. Ltd. (Tokyo:4506; Osaka:4506),

Osaka, Japan

Eisai Co. Ltd. (Tokyo:4523; Osaka:4523), Tokyo, Japan

Ex Scientia Ltd., Dundee, U.K.

Heptares Therapeutics Ltd., Welwyn Garden City, U.K.

Pfizer Inc. (NYSE:PFE), New York, N.Y.

Sunovion Pharmaceuticals Inc., Marlborough, Mass.

University of Dundee, Dundee, U.K.

The University of North Carolina at Chapel Hill School of

Medicine, Chapel Hill, N.C. 